JOURNAL OF INTEGRAL EQUATIONS

AND APPLICATIONS

Volume 17, Number 1, Spring 2005

\title{
HAMILTONIAN EIGENVALUE SYMMETRY FOR QUADRATIC OPERATOR EIGENVALUE PROBLEMS
}

\author{
C. PESTER
}

\begin{abstract}
When the eigenvalues of a given eigenvalue problem are symmetric with respect to the real and the imaginary axes, we speak about a Hamiltonian eigenvalue symmetry or a Hamiltonian structure of the spectrum. This property can be exploited for an efficient computation of the eigenvalues. For some elliptic boundary value problems it is known that the derived eigenvalue problems have this Hamiltonian symmetry. Without having a specific application in mind, we trace the question, under which assumptions the spectrum of a given quadratic eigenvalue problem possesses the Hamiltonian structure.
\end{abstract}

1. Introduction. Nonlinear eigenvalue problems arise in several fields of mathematics including the analysis and numerical solution of higher order systems of ordinary differential equations, for example, in the robot model with electric motors in the joints, see [15] or [12]. Although solvers for quadratic eigenvalue problems are known, see, for example, $[\mathbf{4}]$, the common way is to linearize the eigenvalue problem and to transform it into a standard eigenvalue problem, for whose efficient solution many algorithms exist; a good summary is given by Watkins [16].

We focus on polynomial eigenvalue problems of order two. Quadratic eigenvalue problems are treated, for instance, in the analysis and algebra communities as well as in the engineering community. Depending on the underlying model problem, the resulting quadratic operator eigenvalue problem might have a considerable structure which can be exploited in the further analysis and computations. Our numerical interest in a detailed analysis of such a structure arose from papers including work by Leguillon [10], Kozlov, Maz'ya and Roßmann [9], Apel, Sändig and Solov'ev [3] and Apel, Mehrmann and Watkins [2],

\footnotetext{
2000 AMS Mathematics Subject Classification. Primary 35P30, 37K05, 65F15.

Key words and phrases. Nonlinear eigenvalue problems, Hamiltonian eigenvalue symmetry, spectral theory, operator pencil.

Received by the editors on November 29, 2004, and in revised form on January 13 , 2005.

Copyright (C)2005 Rocky Mountain Mathematics Consortium
} 


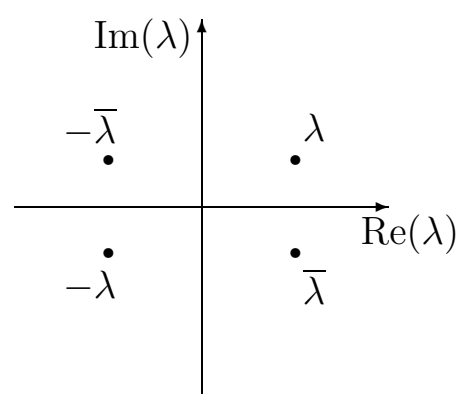

FIGURE 1. The Hamiltonian structure of eigenvalues.

who studied the linear elasticity problem in the neighborhood of polyhedral corners. The computation of the singular part of the solution to the linear elasticity problem near a polyhedral corner involves the solution of a quadratic eigenvalue problem of the form: Find $\lambda \in \mathbf{C}$, $u \in \mathcal{H}$, such that for all $v \in \mathcal{H}$

$$
\lambda^{2} m(u, v)+\lambda g(u, v)=k(u, v),
$$

where $\mathcal{H}$ is a given Hilbert space. The functions $m, g$ and $k$ are sesquilinear forms defined on $\mathcal{H} \times \mathcal{H}$, where $m$ and $k$ are Hermitian and $g$ is skew-Hermitian.

For some elliptic boundary value problems, it is well known that the associated eigenvalue problems have a considerable structure: the eigenvalues appear in pairs $(\lambda,-\lambda)$ if $\lambda$ is real or purely imaginary and in quadruplets $(\lambda,-\lambda, \bar{\lambda},-\bar{\lambda})$ otherwise, see Figure 1 . This property is called Hamiltonian eigenvalue symmetry or Hamiltonian structure of the spectrum, motivated by the spectral properties of a Hamiltonian matrix, see, for example, [12].

The Hamiltonian structure for eigenvalue problems which are associated with certain elliptic boundary value problems in the neighborhood of three-dimensional polyhedral corners was observed, for instance, by Leguillon [10] as a generalization of the two-dimensional case or by Kozlov, Maz'ya and Roßmann [9] (see also references therein) who applied a Mellin transformation to the given boundary value problem. 
Under which assumptions is the Hamiltonian eigenvalue symmetry guaranteed for problem (1), in general? The focus in this paper lies on eigenvalue problems of type (1) without any specific application in the background. Obviously, the Hamiltonian structure is not always given. Consider, for instance, $g(u, v)=\mathrm{i} m(u, v), k(u, v) \equiv 0$, where $\mathrm{i} \in \mathbf{C}$ with $i^{2}=-1$. Then $\lambda=-i$ is an eigenvalue of the problem

$$
\lambda^{2} m(u, v)+\lambda \mathrm{i} m(u, v)=0
$$

but $\lambda=\mathrm{i}$ is not. We traced the spectral properties of problem (1) and formulated a series of conditions which imply the Hamiltonian structure. Since the conditions are formulated in an easy-to-check state, they are sufficient, but not urgently necessary as is demonstrated in some examples in Section 4. Still, they are satisfied in the practical applications that we have in mind.

The Hamiltonian eigenvalue symmetry is of interest in the further analysis of the problem like an efficient solution of its discrete formulation. Adapted Arnoldi and Lanzcos algorithms can be applied then which exploit the underlying structure. For a description of such algorithms, we refer to $[\mathbf{2}, \mathbf{5}, \mathbf{6}, \mathbf{8}, \mathbf{1 1}, \mathbf{1 7}]$.

We could not find a self-contained proof or a summary of conditions for the Hamiltonian structure of the spectrum of a quadratic eigenvalue problem in the linear algebra, functional analysis or numerical analysis literature. In a few pages, we give in this paper an overview of the most important ingredients and definitions, followed by a list of assumptions and a read-on-proof of the Hamiltonian eigenvalue symmetry, including a discussion of the necessity of the assumptions. Of course, other methods exist to prove this result, suggested, for example, by Kozlov, Maz'ya and Roßmann [9], but they usually employ more background information of the given problem and refer to further literature.

As the derivation requires ingredients from several fields of the mathematical sciences, we give an overview of the most important terms in Section 2. For details, we refer to standard work in functional analysis, operator theory, spectral theory, algebra and numerical analysis. In Section 3, we discuss spectral properties of problem (1) and an associated eigenvalue problem for an operator pencil. We conclude the paper with a few examples including an application of the results in Section 4. 


\section{Operators in Hilbert spaces.}

2.1 General notation. Let $H_{R}$ be a (separable) real Hilbert space and denote by $H_{C}$ its complexification

$$
H_{C}=H_{R} \times H_{R}=\left\{u=\left(u_{1}, u_{2}\right) \mid u_{1}, u_{2} \in H_{R}\right\}
$$

with the operations

$$
\begin{aligned}
& \left(u_{1}, u_{2}\right)+\left(v_{1}, v_{2}\right)=\left(u_{1}+v_{1}, u_{2}+v_{2}\right) \quad \forall u_{i}, v_{i} \in H_{R}, \\
& (a+\mathrm{i} b)(u, v)=(a u-b v, b u+a v) \quad \forall a, b \in \mathbf{R}, \quad \forall u, v \in H_{R} .
\end{aligned}
$$

Each element $u_{R} \in H_{R}$ shall be identified with the element $\left(u_{R}, 0\right) \in$ $H_{C}$ so that $H_{R} \subset H_{C}$ and each element $u=\left(u_{1}, u_{2}\right) \in H_{C}$ can be written as $u=\left(u_{1}, 0\right)+\mathrm{i}\left(u_{2}, 0\right)=u_{1}+\mathrm{i} u_{2}$. Moreover, we define the conjugate of $u=\left(u_{1}, u_{2}\right) \in H_{C}$ by

$$
\bar{u}=\overline{u_{1}+\mathrm{i} u_{2}}=u_{1}-\mathrm{i} u_{2} .
$$

Definition 2.2 (Sesquilinear form). A map $a: H_{C} \times H_{C} \rightarrow \mathbf{C}$ is called a sesquilinear form over $H_{C}$, if

$$
a(\mu u, v)=\mu a(u, v), \quad a(u, \mu v)=\bar{\mu} a(u, v), \quad \forall \mu \in \mathbf{C}, \quad \forall u, v \in H_{C}
$$

and

$$
\begin{array}{ll}
a(u+w, v)=a(u, v)+a(w, v) & \forall u, v, w \in H_{C} \\
a(u, v+w)=a(u, v)+a(u, w) & \forall u, v, w \in H_{C} .
\end{array}
$$

Let $\langle\cdot, \cdot\rangle_{H_{C}}: H_{C} \times H_{C} \rightarrow \mathbf{C}$ denote an inner product on $H_{C}$, that is a Hermitian, positive definite sesquilinear form. A norm in $H_{C}$ is then given by $\|u\|_{H_{C}}:=\sqrt{\langle u, u\rangle_{H_{C}}}$.

Definition 2.2 (Real operator). A linear, bounded operator $\mathcal{A}$ : $H_{C} \rightarrow H_{C}$ is called a real operator if $\mathcal{A}\left(H_{R}\right) \subset H_{R}$, that is, if $\mathcal{A} u_{R} \in H_{R}$ for all $u_{R} \in H_{R}$. 
Definition 2.3 (Spectrum of an operator pencil). Let $\mathcal{A}: \mathbf{C} \rightarrow$ $\mathcal{L}\left(H_{C}, H_{C}\right)$ be an operator pencil, that is, $\mathcal{A}(\alpha): H_{C} \rightarrow H_{C}$ for fixed $\alpha \in \mathbf{C}$. The set

$$
\Sigma(\mathcal{A}):=\{\alpha \in \mathbf{C} \mid \mathcal{A}(\alpha) \text { has no bounded inverse }\}
$$

is called the spectrum of $\mathcal{A}(\cdot)$. The number $\alpha_{0} \in \Sigma(\mathcal{A})$ is called the eigenvalue of $\mathcal{A}(\cdot)$, if there exists an element $u_{\alpha_{0}} \in H_{C} \backslash\{0\}$ so that $\mathcal{A}(\alpha) u_{\alpha_{0}}=0$. We denote the set of all eigenvalues of $\mathcal{A}(\cdot)$ by $\sigma(\mathcal{A})$ :

$$
\sigma(\mathcal{A}):=\left\{\alpha \in \mathbf{C} \mid \exists u \in H_{C} \backslash\{0\}, \text { so that } \mathcal{A}(\alpha) u=0\right\} .
$$

Eigenvalue problems for operator pencils have already been considered, for example, by Kozlov, Maz'ya and Roßmann [9].

Remark 2.4. We say that an operator $\mathcal{J}: X \rightarrow Y$ is invertible (or that $\mathcal{J}^{-1}$ exists), if for all $y \in Y$ there is a unique element $x \in X$, so that $\mathcal{J} x=y$. In some books, the invertibility is defined by substituting $Y$ by the range $R(\mathcal{J})$ of $\mathcal{J}$; then, the operator $\mathcal{J}$ is surjective by definition and the invertibility is equivalent to the injectivity of $\mathcal{J}$.

Note that the sets $\Sigma(\mathcal{A})$ and $\sigma(\mathcal{A})$ are equal for finite-dimensional operators; but they might differ for operators in infinite-dimensional spaces. The set $\Sigma(\mathcal{A}) \backslash \sigma(\mathcal{A})$ is called the continuous spectrum of $\mathcal{A}(\cdot)$. The equivalence of $\operatorname{ker}(\mathcal{A}(\alpha))=\{0\}$ and the invertibility of $\mathcal{A}(\alpha)$ is given only for special linear operators $\mathcal{A}(\cdot)$, see Section 2.2 .

2.2 Compact operators and Fredholm operators. In this subsection, we give the definitions and, for our purposes, the most important properties of compact and Fredholm operators. For details and proofs, consult $[1,7, \mathbf{1 4}, \mathbf{1 8}]$ or any standard book on functional analysis or operator theory.

Let $H$ be a separable Hilbert space with an inner product $\langle\cdot, \cdot\rangle_{H}$ : $H \times H \rightarrow \mathbf{C}$ on $H$.

Definition 2.5 (Compact operators). A linear, bounded operator $\mathcal{C}: H \rightarrow H$ is called compact if, for each bounded sequence $\left\{x_{n}\right\}$ in $H$, there is a subsequence $\left\{x_{n_{k}}\right\}$ so that $\mathcal{C} x_{n_{k}}$ converges in $H$, that is, if $\left\|x_{n}\right\|_{H}<\infty \quad \forall n \Longrightarrow \exists x_{n_{k}}, y^{\star} \in H:\left\|\mathcal{C} x_{n_{k}}-y^{\star}\right\|_{H} \longrightarrow 0 \quad$ as $k \rightarrow \infty$. 
Remark 2.6. Let $K(H)$ denote the set of all compact operators on $H$. Then $K(H)$ is a subspace of the space $\mathcal{L}(H)$ of all bounded, linear operators on $H$, see [18, Satz II.3.2 (a)]. This means, in particular, that the sum of two compact operators as well as a scalar multiple of a compact operator is in $K(H)$ and therefore compact.

Definition 2.7 (Compact embedding). We say that a space $V$ is compactly embedded into a space $H \supset V$ if the corresponding embedding operator is compact, that is, if for each bounded sequence $\left\{u_{n}\right\}$ in $V$ there is a subsequence $\left\{u_{n_{k}}\right\}$ with strong convergence in $H$.

We denote by $R(\mathcal{A})$ and $\operatorname{ker}(\mathcal{A})$ the range and the kernel of the linear, bounded operator $\mathcal{A}: H \rightarrow H$, respectively, and by $R\left(\mathcal{A}^{\star}\right)$ and $\operatorname{ker}\left(\mathcal{A}^{\star}\right)$ the range and the kernel of its adjoint. The following lemma is a wellknown result in functional analysis, see, for example, [18, Satz III.4.5].

Lemma 2.8. The relations

$$
\operatorname{ker}(\mathcal{A}) \oplus \overline{R\left(\mathcal{A}^{\star}\right)}=H \quad \text { and } \quad \operatorname{ker}\left(\mathcal{A}^{\star}\right) \oplus \overline{R(\mathcal{A})}=H
$$

hold for each linear, bounded operator $\mathcal{A}: H \rightarrow H$.

Definition 2.9 (Fredholm operators). A linear, bounded operator $\mathcal{T}$ is called a Fredholm operator if $R(\mathcal{T})=\overline{R(\mathcal{T})}$ and $\operatorname{dim} \operatorname{ker} \mathcal{T}<\infty$ and $\operatorname{dim} \operatorname{ker} \mathcal{T}^{\star}<\infty$. For a Fredholm operator $\mathcal{T}$, the number Ind $\mathcal{T}:=\operatorname{dim} \operatorname{ker} \mathcal{T}-\operatorname{dim} \operatorname{ker} \mathcal{T}^{\star}$ is called the index of $\mathcal{T}$.

Lemma 2.10. For each linear, bounded operator $\mathcal{T}$ the following properties are equivalent:

(a) $\mathcal{T}$ is Fredholm and $\operatorname{Ind} \mathcal{T}=0$.

(b) There is a compact operator $\mathcal{C}$ and an invertible operator $\mathcal{J}$, so that $\mathcal{T}=\mathcal{J}-\mathcal{C}$.

Remark 2.11. Suppose that (a) is true in Lemma 2.10. Then $\operatorname{dim} \operatorname{ker} \mathcal{T}=\operatorname{dim} \operatorname{ker} \mathcal{T}^{\star}$ and thus $\operatorname{ker} \mathcal{T}=\{0\}$ if and only if $\operatorname{ker} \mathcal{T}^{\star}=$ $\{0\}$. As a consequence of Lemma 2.8, we have that $\overline{R(\mathcal{T})}=H$ if and 
only if $\overline{R\left(\mathcal{T}^{\star}\right)}=H$. Hence, $\mathcal{T}$ is invertible if and only if ker $\mathcal{T}=\{0\}$. Moreover, $\mathcal{T}$ is invertible if and only if $\mathcal{T}^{\star}$ is invertible.

\section{The quadratic eigenvalue problem.}

3.1 Definitions and assumptions. Let $m, g, k: H_{C} \times H_{C} \rightarrow \mathbf{C}$ be sesquilinear forms over the Hilbert space $H_{C}$ with

$$
\begin{gathered}
k(u, v)=\overline{k(v, u)}, \quad g(u, v)=-\overline{g(v, u)}, \quad m(u, v)=\overline{m(v, u)} \\
\forall u, v \in H_{C} .
\end{gathered}
$$

We consider the quadratic eigenvalue problem (1): Find $\lambda \in \mathbf{C}$, $u \in H_{C} \backslash\{0\}$, such that

$$
\lambda^{2} m(u, v)+\lambda g(u, v)=k(u, v) \quad \forall v \in H_{C} .
$$

A solution $(\lambda, u) \in \mathbf{C} \times\left(H_{C} \backslash\{0\}\right)$ to problem (1) is called an eigenpair of problem (1); the number $\lambda$ is then called eigenvalue and $u$ is called eigenelement.

Definition 3.1 (Hamiltonian eigenvalue symmetry). We say that the eigenvalue problem (1) possesses a Hamiltonian eigenvalue symmetry or that its spectrum has a Hamiltonian structure, if the eigenvalues are placed symmetric with respect to the real and the imaginary axes; that is, if $\lambda \in \mathbf{C}$ is an eigenvalue of problem (1), then so are $-\lambda, \bar{\lambda},-\bar{\lambda}$.

Remark 3.2. A real two-by-two block matrix $H$ is called Hamiltonian if

$$
(J H)^{\top}=J H \quad \text { with } \quad J=\left(\begin{array}{cc}
O & I \\
-I & O
\end{array}\right) .
$$

The term Hamiltonian eigenvalue symmetry is motivated by the structure of the spectrum of Hamiltonian matrices.

The question is under which conditions on $m, g, k$ and $H_{C}$ the Hamiltonian eigenvalue symmetry is given. In the derivation of the desired properties, we followed the ideas of $[\mathbf{3}]$, where a quadratic eigenvalue problem of type (1) is treated and transformed into an eigenvalue problem for an operator pencil. 
In the following assumptions, we summarize conditions that are sufficient for the Hamiltonian structure. They are usually easy to check and satisfied in the applications that we have in mind.

(I) $V:=H_{C}:=H_{R} \times H_{R}$ is the complexification of a separable real Hilbert space $H_{R}$, and $H \supset V$ is a Hilbert space into which $V$ is compactly embedded.

(II) The functions $m: H \times H \rightarrow \mathbf{C}, g, k: V \times V \rightarrow \mathbf{C}$ are sesquilinear forms with

$$
m(u, v)=\overline{m(v, u)} \quad \forall u, v \in H, \quad k(u, v)=\overline{k(v, u)} \quad \forall u, v \in V
$$

(III) The functions $m, g, k$ are real, that is,

$$
\begin{gathered}
m(\bar{u}, \bar{v})=\overline{m(u, v)}, \quad g(\bar{u}, \bar{v})=\overline{g(u, v)}, \quad k(\bar{u}, \bar{v})=\overline{k(u, v)} \\
\forall u, v \in V .
\end{gathered}
$$

(IV) The sesquilinear form $m$ satisfies

$$
|m(u, v)| \leq c\|u\|_{H}\|v\|_{H} \quad \forall u \in H .
$$

(V) There is a sesquilinear form $d: H \times V \rightarrow \mathbf{C}$ so that $g(u, v)=$ $\overline{d(v, u)}-d(u, v)$ for all $u, v \in V$ and

$$
|d(u, v)| \leq c\|u\|_{H}\|v\|_{V} \quad \forall u, v \in V .
$$

(VI) The operator $\mathcal{K}: V \rightarrow V$ induced by $k(u, v)=\langle\mathcal{K} u, v\rangle_{V}$ for all $u, v \in V$ is invertible; and for each fixed element $u \in H$, there is a constant $c_{k}>0$, so that $|k(u, v)| \leq c_{k}\|v\|_{V}$ for all $v \in V$.

Alternatively, it is sufficient that the sesquilinear form $k$ is positive definite, that is,

$$
k(u, u) \geq 0 \quad \forall u \in V, \quad k(u, u)=0 \Longleftrightarrow u=0
$$

In the following, $V$ and $H$ denote the Hilbert spaces introduced by assumption (I). 
Remark 3.3. The condition (III) that the functions $m, g$ and $k$ are real does not mean that they are real-valued, but only that they have real values for real arguments. This is equivalent to

$$
m\left(u_{R}, v_{R}\right) \in \mathbf{R}, \quad g\left(u_{R}, v_{R}\right) \in \mathbf{R}, \quad k\left(u_{R}, v_{R}\right) \in \mathbf{R}, \quad \forall u_{R}, v_{R} \in H_{R} .
$$

Given a skew-Hermitian sesquilinear form $g: V \times V \rightarrow \mathbf{C}$, there is always a map $d: V \times V \rightarrow \mathbf{C}$ so that $g(u, v)=\overline{d(v, u)}-d(u, v)$, for example, $d(u, v)=-(1 / 2) g(u, v)$. Assumption (V), however, demands that the sesquilinear form $d$ is defined on a larger space, on $H \times V$, so that $d=-(1 / 2) g$ is not necessarily the desired function. That is why we cannot simply omit $d$ or $g$. Still, the function $g$ has to be defined only on the space $V \times V$, whereas $m$ has the largest domain $H \times H$ due to assumption (II).

If $k(\cdot, \cdot)$ defines an inner product on $V$, that is, if $k(\cdot, \cdot)$ is positive definite, we can choose $\langle u, v\rangle_{V}=k(u, v)$ for all $u, v \in V$. Then, the operator $\mathcal{K}$ in condition (VI) equals the identity operator $\mathcal{I}$ on $V$ and is therefore invertible.

For the inner products $\langle\cdot, \cdot\rangle_{V}$ in $V$ and $\langle\cdot, \cdot\rangle_{H}$ in $H$, the corresponding norms are given by $\|u\|_{V}^{2}=\langle u, u\rangle_{V}$ and $\|u\|_{H}^{2}=\langle u, u\rangle_{H}$. Due to assumption (I), the space $V$ is embedded into $H$. Thus, the relation

$$
\|u\|_{H} \leq c\|u\|_{V}
$$

holds for all $u \in V$, where $c$ is a generic constant independent of $u$.

In the remaining section, we prove that the Hamiltonian eigenvalue symmetry is actually given under these assumptions. When a weaker condition is sufficient in one or another step, we will point this out.

3.2 The eigenvalue problem for an operator pencil. Corresponding to the sesquilinear forms $m, g$ and $k$, we introduce the linear operators $\mathcal{M}, \mathcal{G}, \mathcal{K}: V \rightarrow V$ by

$$
\begin{array}{lcrl}
\mathcal{M} u \in V, & \langle\mathcal{M} u, v\rangle_{V}=m(u, v) & \forall v \in V, \\
\mathcal{G} u \in V, & \langle\mathcal{G} u, v\rangle_{V}=g(u, v) & \forall v \in V, \\
\mathcal{K} u \in V, & \langle\mathcal{K} u, v\rangle_{V}=k(u, v) & \forall v \in V .
\end{array}
$$


The adjoint operators $\mathcal{M}^{\star}, \mathcal{G}^{\star}$ and $\mathcal{K}^{\star}$ are given by

$$
\begin{aligned}
\langle\mathcal{M} u, v\rangle_{V} & =\left\langle u, \mathcal{M}^{\star} v\right\rangle_{V} & & \forall u, v \in V, \\
\langle\mathcal{G} u, v\rangle_{V} & =\left\langle u, \mathcal{G}^{\star} v\right\rangle_{V} & & \forall u, v \in V, \\
\langle\mathcal{K} u, v\rangle_{V} & =\left\langle u, \mathcal{K}^{\star} v\right\rangle_{V} & & \forall u, v \in V .
\end{aligned}
$$

Lemma 3.4. The operators $\mathcal{M}, \mathcal{G}$ and $\mathcal{K}$ are well-defined.

Proof. The Riesz representation theorem states: Let $\langle\cdot, \cdot\rangle_{X}$ be an inner product on a Hilbert space $X$. Then for each bounded linear functional $F \in X^{\star}$, there is a unique element $u_{F} \in X$, so that $\left\langle v, u_{F}\right\rangle_{X}=F(v)$ for all $v \in X$, see, for example, [1].

We choose $X:=V$ and $\langle\cdot, \cdot\rangle_{X}:=\langle\cdot, \cdot\rangle_{V}$ as well as $F(v):=F_{m, u_{0}}(v):=$ $\overline{m\left(u_{0}, v\right)}$ for a fixed element $u_{0} \in V$. Indeed, $F_{m, u_{0}}(v)$ is a linear functional; but, for its boundedness, we have to assume that $\left|m\left(u_{0}, v\right)\right| \leq c\|v\|_{V}$ for all $v \in V$ and for each fixed $u_{0} \in V$ with a generic constant $c$ independent of $v$. Then, there is a unique element $u_{F_{m, u_{0}}} \in V$, so that $\left\langle v, u_{F_{m, u_{0}}}\right\rangle_{V}=\overline{m\left(u_{0}, v\right)}$, and therefore, $\left\langle u_{F_{m, u_{0}}}, v\right\rangle_{V}=m\left(u_{0}, v\right)$ for all $v \in V$. Consequently, $\mathcal{M} u_{0}$ is given by $\mathcal{M} u_{0}=u_{F_{m, u_{0}}}$.

Analogously, there exist unique elements $u_{F_{g, u_{0}}}, u_{F_{k, u_{0}}} \in V$, so that $\mathcal{G} u_{0}=u_{F_{g, u_{0}}}$ and $\mathcal{K} u_{0}=u_{F_{k, u_{0}}}$, respectively, if we assume that $\left|g\left(u_{0}, v\right)\right| \leq c\|v\|_{V}$ and $\left|k\left(u_{0}, v\right)\right| \leq c\|v\|_{V}$ for all $v \in V$ and for each fixed $u_{0} \in V$.

These boundedness conditions on $m, g$ and $k$ are satisfied thanks to assumptions (IV), (V), (VI) and the embedding property (2).

Lemma 3.5. The operators satisfy $\mathcal{M}^{\star}=\mathcal{M}, \mathcal{G}^{\star}=-\mathcal{G}, \mathcal{K}^{\star}=\mathcal{K}$.

Proof. The assertion follows from

$$
\begin{aligned}
\langle\mathcal{M} u, v\rangle_{V} & =m(u, v)=\overline{m(v, u)}=\overline{\langle\mathcal{M} v, u\rangle_{V}} \\
& =\overline{\left\langle v, \mathcal{M}^{\star} u\right\rangle_{V}}=\left\langle\mathcal{M}^{\star} u, v\right\rangle_{V} \\
\langle\mathcal{G} u, v\rangle_{V} & =g(u, v)=-\overline{g(v, u)}=-\overline{\langle\mathcal{G} v, u\rangle_{V}} \\
& =-{\overline{\left\langle v, \mathcal{G}^{\star} u\right\rangle_{V}}}=\left\langle-\mathcal{G}^{\star} u, v\right\rangle_{V}
\end{aligned}
$$




$$
\begin{aligned}
& \langle\mathcal{K} u, v\rangle_{V}=k(u, v)=\overline{k(v, u)}={\overline{\langle\mathcal{K} v, u\rangle_{V}}} \\
& ={\overline{\left\langle v, \mathcal{K}^{\star} u\right\rangle_{V}}}=\left\langle\mathcal{K}^{\star} u, v\right\rangle_{V} .
\end{aligned}
$$

From the definition of $\mathcal{M}^{\star}, \mathcal{G}^{\star}, \mathcal{K}^{\star}$, we conclude that $\mathcal{M}^{\star \star}=\mathcal{M}$, $\mathcal{G}^{\star \star}=\mathcal{G}, \mathcal{K}^{\star \star}=\mathcal{K}$, since $\langle\mathcal{M} u, v\rangle_{V}=\left\langle u, \mathcal{M}^{\star} v\right\rangle_{V}=\overline{\left\langle\mathcal{M}^{\star} v, u\right\rangle_{V}}=$ ${\overline{\left\langle v, \mathcal{M}^{\star \star} u\right\rangle_{V}}}=\left\langle\mathcal{M}^{\star \star} u, v\right\rangle_{V}$ and, analogously, $\langle\mathcal{G} u, v\rangle_{V}=\left\langle\mathcal{G}^{\star \star} u, v\right\rangle_{V}$ and $\langle\mathcal{K} u, v\rangle_{V}=\left\langle\mathcal{K}^{\star \star} u, v\right\rangle_{V}$ for all $u, v \in V$.

We introduce the operator pencil $\mathcal{B}(\cdot): \mathbf{C} \rightarrow \mathcal{L}(V, V)$ by

$$
\mathcal{B}(\lambda):=\mathcal{K}-\lambda \mathcal{G}-\lambda^{2} \mathcal{M}, \quad \lambda \in \mathbf{C}
$$

and define the adjoint operator pencil

$$
\mathcal{B}^{\star}(\lambda):=[\mathcal{B}(\lambda)]^{\star}=\mathcal{K}^{\star}-\bar{\lambda} \mathcal{G}^{\star}-\bar{\lambda}^{2} \mathcal{M}^{\star}, \quad \lambda \in \mathbf{C} .
$$

Lemma 3.6 (Operator eigenvalue problem). The quadratic eigenvalue problem (1) is equivalent to the operator eigenvalue problem for $\mathcal{B}(\cdot)$. Find $\lambda \in \mathbf{C}, u \in V \backslash\{0\}$, such that

$$
\mathcal{B}(\lambda) u=0 .
$$

Proof. We know from the definition of $\mathcal{M}, \mathcal{G}$ and $\mathcal{K}$ that

$$
\begin{aligned}
\langle\mathcal{B}(\lambda) u, v\rangle_{V} & =\left\langle\left(\mathcal{K}-\lambda \mathcal{G}-\lambda^{2} \mathcal{M}\right) u, v\right\rangle_{V} \\
& =\langle\mathcal{K} u, v\rangle_{V}-\lambda\langle\mathcal{G} u, v\rangle_{V}-\lambda^{2}\langle\mathcal{M} u, v\rangle_{V} \\
& =k(u, v)-\lambda g(u, v)-\lambda^{2} m(u, v) .
\end{aligned}
$$

Furthermore, $(\lambda, u) \in \mathbf{C} \times V$ is an eigenpair of problem (1) if and only if $k(u, v)-\lambda g(u, v)-\lambda^{2} m(u, v)=0$ for all $v \in V$, that is, $\langle\mathcal{B}(\lambda) u, v\rangle_{V}=0$ for all $v \in V$. This is equivalent to $\mathcal{B}(\lambda) u=0$, since $\langle\cdot, \cdot\rangle_{V}$ is positive definite, and therefore, $(\lambda, u)$ is an eigenpair of problem (4).

The assumptions (IV), (V) and (VI) were made so that the operators $\mathcal{M}$ and $\mathcal{G}$ are compact (we will prove this in Section 3.3) and that the 
operator $\mathcal{K}$ is invertible. It follows from Lemma 2.10 and Remark 2.6 that the operator $\mathcal{B}(\lambda)$ is a Fredholm operator with index $\operatorname{Ind} \mathcal{B}(\lambda)=0$ for each $\lambda \in \mathbf{C}$.

Due to Remark 2.11, we know that $\mathcal{B}(\lambda)$ is invertible if and only if the equation $\mathcal{B}(\lambda) u=0$ has only the trivial solution $u=0$. Hence, we can write

$$
\begin{aligned}
\sigma(\mathcal{B}) & =\{\lambda \in \mathbf{C} \mid \exists u \in V \backslash\{0\}, \text { so that } \mathcal{B}(\lambda) u=0\} \\
& =\{\lambda \in \mathbf{C} \mid \mathcal{B}(\lambda) \text { is not invertible }\}
\end{aligned}
$$

3.3 Compactness of $\mathcal{M}$ and $\mathcal{G}$. The aim of this subsection is the verification that the operators $\mathcal{M}$ and $\mathcal{G}$ are compact under the assumptions (I), (IV) and (V).

We consider any bounded sequence $\left\{u_{n}\right\}$ in $V$. Since $V$ is compactly embedded into $H$ due to assumption (I), there is a subsequence $u_{n_{k}}$ which converges in $H$. For the sake of simplicity of notation, we denote this subsequence by $\left\{u_{n}\right\}$ and omit the subindex $k$. We conclude that $\left\{u_{n}\right\}$ is a Cauchy sequence in $H$. Assumption (IV) and the embedding property (2) imply that

$$
\begin{aligned}
\left\|\mathcal{M} u_{n}-\mathcal{M} u_{m}\right\|_{V}^{2} & =\left|\left\langle\mathcal{M} u_{n}-\mathcal{M} u_{m}, \mathcal{M} u_{n}-\mathcal{M} u_{m}\right\rangle_{V}\right| \\
& =\left|m\left(u_{n}-u_{m}, \mathcal{M} u_{n}-\mathcal{M} u_{m}\right)\right| \\
& \leq c\left\|u_{n}-u_{m}\right\|_{H} \cdot\left\|\mathcal{M} u_{n}-\mathcal{M} u_{m}\right\|_{H} \\
& \leq c\left\|u_{n}-u_{m}\right\|_{H} \cdot\left\|\mathcal{M} u_{n}-\mathcal{M} u_{m}\right\|_{V} .
\end{aligned}
$$

Division by $\left\|\mathcal{M} u_{n}-\mathcal{M} u_{m}\right\|_{V}$ yields

$$
\left\|\mathcal{M} u_{n}-\mathcal{M} u_{m}\right\|_{V} \leq c\left\|u_{n}-u_{m}\right\|_{H} .
$$

Since $\left\{u_{n}\right\}$ is a Cauchy sequence in $H$, we obtain that $\left\{\mathcal{M} u_{n}\right\}$ is a Cauchy sequence in $V$. Exploiting that $V$ is complete, we conclude that $\left\{\mathcal{M} u_{n}\right\}$ converges in $V$, which proves the compactness of $\mathcal{M}$.

By analogy with the operator $\mathcal{M}$, we introduce the operator $\mathcal{D}: V \rightarrow$ $V$ by

$$
\langle\mathcal{D} u, v\rangle_{V}=d(u, v) \quad \forall u, v \in V .
$$


Then, with the same arguments as above, we get from assumption (V) that the operator $\mathcal{D}$ is compact. Hence, the adjoint operator $\mathcal{D}^{\star}$, which is given by

$$
\langle\mathcal{D} u, v\rangle_{V}=\left\langle u, \mathcal{D}^{\star} v\right\rangle_{V} \quad \forall u, v \in V,
$$

is compact as well (theorem of Schauder), see [7]. Moreover, we have for all $u, v \in V$ that

$$
\begin{aligned}
\langle\mathcal{G} u, v\rangle_{V} & =g(u, v)=\overline{d(v, u)}-d(u, v)=\overline{\langle\mathcal{D} v, u\rangle_{V}}-\langle\mathcal{D} u, v\rangle_{V} \\
& =\overline{\left\langle v, \mathcal{D}^{\star} u\right\rangle_{V}}-\langle\mathcal{D} u, v\rangle_{V}=\left\langle\mathcal{D}^{\star} u, v\right\rangle_{V}-\langle\mathcal{D} u, v\rangle_{V} \\
& =\left\langle\left(\mathcal{D}^{\star}-\mathcal{D}\right) u, v\right\rangle_{V} .
\end{aligned}
$$

Therefore, by Remark 2.6, the operator $\mathcal{G}=\mathcal{D}^{\star}-\mathcal{D}$ is compact.

3.4 Proof: $\lambda \in \sigma(\mathcal{B}) \Rightarrow-\bar{\lambda} \in \sigma(\mathcal{B})$. Returning to the eigenvalue problem for the operator pencil $\mathcal{B}(\cdot)$ and the Hamiltonian structure of the spectrum, we start with the proof that with $\lambda \in \mathbf{C}$ also $-\bar{\lambda} \in \mathbf{C}$ is an eigenvalue of problem (4).

Lemma 3.7. The relation $\mathcal{B}^{\star}(\lambda)=\mathcal{B}(-\bar{\lambda})$ is true for all $\lambda \in \mathbf{C}$.

Proof. Each $\lambda \in \mathbf{C}$ satisfies $\mathcal{B}^{\star}(\lambda)=\mathcal{K}^{\star}-\bar{\lambda} \mathcal{G}^{\star}-\bar{\lambda}^{2} \mathcal{M}^{\star}=\mathcal{K}-(-\bar{\lambda}) \mathcal{G}-$ $(-\bar{\lambda})^{2} \mathcal{M}=\mathcal{B}(-\bar{\lambda})$.

Lemma 3.8. The operators $\mathcal{B}$ and $\mathcal{B}^{\star}$ have the same eigenvalues, that is, $\sigma(\mathcal{B})=\sigma\left(\mathcal{B}^{\star}\right)$.

Proof. Since $\mathcal{B}(\lambda)$ is a Fredholm operator with index 0 , we have that $\lambda \in \sigma(\mathcal{B})$ if and only if $\mathcal{B}(\lambda)$ is not invertible. By Remark 2.11, this is true if and only if $\mathcal{B}^{\star}(\lambda)$ is not invertible, which is equivalent to $\lambda \in \sigma\left(\mathcal{B}^{\star}\right)$.

Corollary 3.9. If $\lambda \in \sigma(\mathcal{B})$, then $-\bar{\lambda} \in \sigma(\mathcal{B})$.

It remains to show either that $\bar{\lambda} \in \sigma(\mathcal{B})$ or that $-\lambda \in \sigma(\mathcal{B})$ given $\lambda \in \sigma(\mathcal{B})$. The existence of the fourth eigenvalue that completes the Hamiltonian structure follows then immediately from Corollary 3.9. 
3.5 Proof: $\lambda \in \sigma(\mathcal{B}) \Rightarrow \bar{\lambda} \in \sigma(\mathcal{B})$. The essential condition for this subsection is that the sesquilinear forms are real, see assumption (III). We use this property to evaluate the term $\overline{\mathcal{B}(\lambda) u}$.

Lemma 3.10. The relations $\overline{\mathcal{M} u}=\mathcal{M} \bar{u}, \overline{\mathcal{G} u}=\mathcal{G} \bar{u}, \overline{\mathcal{K} u}=\mathcal{K} \bar{u}$ are true for all $u \in V$.

Proof. Assumption (III) implies for each $u \in V$ that

$$
\langle\overline{\mathcal{M} u}, v\rangle_{V}=\overline{\langle\mathcal{M} u, \bar{v}\rangle_{V}}=\overline{m(u, \bar{v})}=m(\bar{u}, v)=\langle\mathcal{M} \bar{u}, v\rangle_{V} \quad \forall v \in V,
$$

which proves the assertion for $\overline{\mathcal{M} u}$. The relations $\overline{\mathcal{G} u}=\mathcal{G} \bar{u}$ and $\overline{\mathcal{K} u}=\mathcal{K} \bar{u}$ follow analogously.

Corollary 3.11. The relation $\overline{\mathcal{B}(\lambda) u}=\mathcal{B}(\bar{\lambda}) \bar{u}$ is true for all $u \in V$.

Proof. Lemma 3.10 yields for each $u \in V$ that

$$
\begin{aligned}
\overline{\mathcal{B}(\lambda) u} & =\overline{\left(\mathcal{K}-\lambda \mathcal{G}-\lambda^{2} \mathcal{M}\right) u}=\overline{\mathcal{K} u}-\overline{\lambda \mathcal{G} u}-\overline{\lambda^{2} \mathcal{M} u} \\
& =\mathcal{K} \bar{u}-\bar{\lambda} \mathcal{G} \bar{u}-\bar{\lambda}^{2} \mathcal{M} \bar{u}=\mathcal{B}(\bar{\lambda}) \bar{u} .
\end{aligned}
$$

Corollary 3.12. If $\lambda \in \mathbf{C}$ is an eigenvalue of $\mathcal{B}(\cdot)$ then so is $\bar{\lambda}$.

Proof. If $\lambda \in \mathbf{C}$ is an eigenvalue of $\mathcal{B}(\cdot)$, then there is an element $u \in V \backslash\{0\}$, so that $\mathcal{B}(\lambda) u=0$. Consequently, we have that $\overline{\mathcal{B}(\lambda) u}=0$. Corollary 3.11 implies that $\mathcal{B}(\bar{\lambda}) \bar{u}=0$, that is, $\bar{\lambda}$ is an eigenvalue of $\mathcal{B}(\cdot)$, since $\bar{u} \in V \backslash\{0\}$.

This part of the proof holds even if $\mathcal{B}(\lambda)$ is not Fredholm; this means that the compact embedding part of assumption (I) and the special structure of $g$ formulated in assumption (V) were not used here. Neither was condition (II) employed. The boundedness conditions in (IV)-(VI), however, cannot be omitted, because we used them in the proof that the operators $\mathcal{M}, \mathcal{G}$ and $\mathcal{K}$ are well defined, see Lemma 3.4. 
3.6. The Hamiltonian eigenvalue symmetry. The following theorem is the consequence of the previous results.

Theorem 3.13 (Hamiltonian eigenvalue symmetry). If the assumptions (I)-(VI) are satisfied, then, the spectrum of the quadratic eigenvalue problem (1),

$$
\lambda^{2} m(u, v)+\lambda g(u, v)=k(u, v),
$$

is symmetric with respect to the real and imaginary axes, that is, if $\lambda \in \mathbf{C}$ is an eigenvalue of problem (1), then so are $-\lambda, \bar{\lambda},-\bar{\lambda}$.

The theorem is easily proven by the use of Corollary $3.9(\lambda \in \sigma(\mathcal{B})$ $\Rightarrow-\bar{\lambda} \in \sigma(\mathcal{B}))$, Corollary $3.12(\lambda \in \sigma(\mathcal{B}) \Rightarrow \bar{\lambda} \in \sigma(\mathcal{B}))$ and Lemma 3.6 which states that $\lambda \in \mathbf{C}$ is an eigenvalue of problem (1) if and only if $\mathcal{B}(\lambda) u=0$, where $u \neq 0$ is the corresponding eigenelement.

Remark 3.14. The same symmetry is given for nonlinear eigenvalue problems of higher order,

$$
\sum_{i=0}^{n} \lambda^{i} m_{i}(u, v)=0,
$$

with alternating sequences of Hermitian and skew-Hermitian sesquilinear forms $m_{i}(\cdot, \cdot)$, if $-m_{0}$ satisfies condition (VI), the other Hermitian forms fulfill condition (IV) and (V) holds for the skew-Hermitian forms. Polynomial eigenvalue problems of this form were treated, for example, by Mehrmann and Watkins [12].

\section{Examples.}

Example 4.1. Consider the counter-example of the introduction: $g(u, v)=\mathrm{i} m(u, v), k(u, v) \equiv 0$. Obviously, $k$ is not positive definite and the operator $\mathcal{K}$ induced by $k(\cdot, \cdot)$ is the zero operator and therefore not invertible. Hence, the Fredholm theory cannot be applied.

Note that, in general, we cannot conclude from this that the desired eigenvalue symmetry is not given; in this example, however, $\lambda=-\mathrm{i}$ is an eigenvalue, but $\lambda=\mathrm{i}$ is not. 
Example 4.2. Let $V$ be a finite-dimensional complex Hilbert space; without loss of generality, $V=\mathbf{C}^{n}, n \in \mathbf{N}$. Let $m, g, k$ be given by

$$
m(u, v)=\bar{v}^{\top} M u, \quad g(u, v)=\bar{v}^{\top} G u, \quad k(u, v)=\bar{v}^{\top} K u \quad \forall u, v \in V,
$$

where $M, G, K$ are complex $n \times n$-matrices.

Let $\langle\cdot, \cdot\rangle_{V}$ denote the usual inner product, $\langle u, v\rangle_{V}:=\bar{v}^{\top} u$. The operator $\mathcal{M}$ induced by $m(u, v)=\langle\mathcal{M} u, v\rangle_{V}$ for all $u, v \in V$ is then defined by $m(u, v)=\bar{v}^{\top} \mathcal{M} u$, that is, $\mathcal{M}=M$. Analogously, we have that $\mathcal{G}=G$ and $\mathcal{K}=K$. Hence, the eigenvalue problem is equivalent to the matrix eigenvalue problem: Find $\lambda \in \mathbf{C}, u \in \mathbf{C}^{n}$, such that

$$
\lambda^{2} M u+\lambda G u=K u \text {. }
$$

The assumptions (II) and (III) imply that $M, G$ and $K$ are real matrices with $M=M^{\top}, G=-G^{\top}$ and $K=K^{\top}$.

Indeed, the Hamiltonian eigenvalue symmetry can be proven then: Assuming that $\lambda$ is an eigenvalue, we get by conjugating and transposing the equation that $-\bar{\lambda}$ is an eigenvalue, too. The characteristic polynomial $\operatorname{det}\left(\lambda^{2} M+\lambda G-K\right)$ has real coefficients, which implies that $\bar{\lambda}$ is an eigenvalue as well, and therefore so is $-\lambda$.

Note that (VI), demanding the invertibility of $K$, need not be satisfied here; nor are (IV) or (V) essential. Nevertheless, it is necessary that $M$, $G$ and $K$ are real. Indeed, one easily finds complex counter-examples, compare $M=I, G=2 \mathrm{i} I, K=2 I$, where $\lambda=-\mathrm{i}+1$ and $-\bar{\lambda}=-\mathrm{i}-1$ are eigenvalues, but $\bar{\lambda}$ and $-\lambda$ are not.

Finally, let us consider a quadratic eigenvalue problem that arises in numerical practice. In the neighborhood of polyhedral corners, the singular part of the solution to the linear elasticity problem has a special structure. The computation of the corner singularities can be reduced to a quadratic eigenvalue problem of type (1), which we learnt from [10]. Since the underlying problem is an elliptic boundary value problem, Kozlov, Maz'ya and Roßmann [9] suggested to apply a Mellin transformation from which the Hamiltonian eigenvalue structure can be obtained as well. Further details including the operator eigenvalue problem formulated in Section 3.2 are given by Apel, Sändig and Solov'ev [3]. 
Example 4.3. The study of corner singularities leads to the consideration of a ball circumscribing the polyhedral corner. Using spherical coordinates and a specific approach for the structure of the solution to the linear elasticity problem, one obtains the quadratic eigenvalue problem

$$
\lambda^{2} m(u, v)+\lambda g(u, v)=k(u, v),
$$

see [13] with $\lambda \in \mathbf{C}, u, v \in V$, where $V=H_{0}^{1}(\Omega)^{3}$ and $H=L^{2}(\Omega)^{3}$ are spaces of vector functions over a subdomain $\Omega$ of the unit sphere in $\mathbf{R}^{3}$. This eigenvalue problem was written down in a slightly different form by Leguillon [10]; we refer also to the paper by Apel, Sändig and Solov'ev [3], which was the basis for the derivation of the Hamiltonian eigenvalue symmetry in this paper.

The space $H_{0}^{1}(\Omega)$ is compactly embedded into $L^{2}(\Omega)$, hence so is $V$ into $H$. The assumptions on $m, g$ and $k$ are satisfied in the given problem; therefore, the suggested symmetry of the spectrum can be concluded, as was done rather shortly by Apel, Sändig and Solov'ev [3, Theorem 10] or Leguillon [10] or only for the discrete formulation, for instance, by Apel, Mehrmann and Watkins [2, Proposition 1], see also Mehrmann and Watkins [12] and references therein.

5. Summary. We studied a quadratic eigenvalue problem and formulated conditions under which the Hamiltonian eigenvalue symmetry can be proven. To derive the Hamiltonian structure, we transformed the problem into an eigenvalue problem for an operator pencil and applied Fredholm theory. Since it is usually difficult to check whether a given operator is a Fredholm operator with index 0 or not, we formulated simplified conditions in (I)-(VI) which imply the Fredholm property. At last, we proved that under these assumptions the spectrum of the quadratic eigenvalue problem

$$
\lambda^{2} m(u, v)+\lambda g(u, v)=k(u, v)
$$

has the desired structure. In general, these conditions are easy to check, once the functions $m, g, k$ are given.

Finally, we presented an application and discussed the necessity of the assumptions in two further examples. 
Acknowledgments. For fruitful discussion and valuable advice, special thanks go to Thomas Apel (Universität der Bundeswehr München), Bernd Silbermann (Technische Universität Chemnitz) and Sergey Solov'ev (Kazan State University). We also thank the DFG (Deutsche Forschungsgemeinschaft) for financial support.

\section{REFERENCES}

1. H.W. Alt, Lineare Funktionalanalysis, Springer, Berlin, 3. Auflage, 1999.

2. T. Apel, V. Mehrmann and D. Watkins, Structured eigenvalue methods for the computation of corner singularities in $3 \mathrm{D}$ anisotropic elastic structures, Comput. Methods Appl. Mech. Engrg. 191 (2002), 4459-4473.

3. T. Apel, A.M. Sändig and S.I. Solov'ev, Computation of 3d vertex singularities for linear elasticity: Error estimates for a finite element method on graded meshes, Math. Model. Numer. Anal. 36 (2002), 1043-1070.

4. Z. Bai, Krylov subspace techniques for reduced-order modeling of large-scale dynamical systems, Appl. Numer. Math. 43 (2002), 9-44.

5. P. Benner and H. Faßbender, An implicitly restarted symplectic Lanczos method for the Hamiltonian eigenvalue problem, Linear Alg. Appl. 263 (1997), $75-111$.

6. P. Benner and H. Faßbender, An implicitly restarted symplectic Lanczos method for the symplectic eigenvalue problem, SIAM J. Matrix Anal. Appl. 22 (2000), 682-713.

7. M. Cotlar and R. Cignoli, An introduction to functional analysis, NorthHolland Publ. Co., Amsterdam.

8. R.W. Freund, Lanczos-type algorithms for structured non-Hermitian eigenvalue problems, Proc. of the Cornelius Lanczos Internat. Centenary Conf., SIAM, Philadelphia, 1994, pp. 243-245.

9. V.A. Kozlov, V.G. Maz'ya and J. Roßmann, Spectral problems associated with corner singularities of solutions to elliptic equations, Math. Surveys Monographs, vol. 85, Amer. Math. Soc., 2000.

10. D. Leguillon, Computation of $3 d$ singularities in elasticity, in Boundary value problems and integral equations in nonsmooth domains, Proc. of Conf. at CIRM (Luminy, France, May 3-7, 1993), Lecture Notes in Pure Appl. Math., vol. 167, Marcel Dekker, New York, 1995, pp. 161-170.

11. V. Mehrmann and D. Watkins, Structure-preserving methods for computing eigenpairs of large sparse skew-Hamiltonian/Hamiltonian pencils, SIAM J. Sci. Statist. Comput. 22 (2002), 1905-1925.

12. — Polynomial eigenvalue problems with Hamiltonian structure, Electron. Trans. Numer. Anal. 13 (2002), 106-118.

13. A. Meyer and C. Pester, The Laplace and the linear elasticity problems near polyhedral corners and associated eigenvalue problems, Tech. Rep. SFB393/04-12, 2004.

14. S. Prößdorf, Einige Klassen singulärer Gleichungen, Akademie-Verlag, Berlin. 
15. W. Schiehlen, Multibody systems handbook, Springer-Verlag, Berlin, 1990.

16. D. Watkins, Fundamentals of matrix computations, Wiley, New York, 2002.

17. - On Hamiltonian and symplectic Lanczos processes, Linear Algebra Appl. 385 (2004), 23-45.

18. D. Werner, Funktionalanalysis, Springer, Berlin, 2. Auflage, 1997.

Institut FÜr MAthematik und Bauinformatik, Universität Der BunDeswehr München, 85577 Neubiberg, Germany

E-mail address: cornelia.pester@unibw-muenchen.de 